\title{
Modified Quasi-Steady State Model of DC System for Transient Stability Simulation under Asymmetric Faults
}

\author{
Jun Liu, ${ }^{1}$ Zhanhong Wei, ${ }^{1}$ Wanliang Fang, ${ }^{1}$ Chao Duan, ${ }^{1}$ Junxian Hou, ${ }^{2}$ and Zutao Xiang ${ }^{2}$ \\ ${ }^{1}$ State Key Laboratory of Electrical Insulation and Power Equipment, School of Electrical Engineering, Xian Jiaotong University, \\ Xian, Shaanxi 710049, China \\ ${ }^{2}$ China Electric Power Research Institute, Haidian District, Beijing 100085, China
}

Correspondence should be addressed to Wanliang Fang; eewlfang@mail.xjtu.edu.cn

Received 25 May 2015; Accepted 15 July 2015

Academic Editor: Xiaosong Hu

Copyright (C) 2015 Jun Liu et al. This is an open access article distributed under the Creative Commons Attribution License, which permits unrestricted use, distribution, and reproduction in any medium, provided the original work is properly cited.

\begin{abstract}
As using the classical quasi-steady state (QSS) model could not be able to accurately simulate the dynamic characteristics of DC transmission and its controlling systems in electromechanical transient stability simulation, when asymmetric fault occurs in AC system, a modified quasi-steady state model (MQSS) is proposed. The model firstly analyzes the calculation error induced by classical QSS model under asymmetric commutation voltage, which is mainly caused by the commutation voltage zero offset thus making inaccurate calculation of the average DC voltage and the inverter extinction advance angle. The new MQSS model calculates the average DC voltage according to the actual half-cycle voltage waveform on the DC terminal after fault occurrence, and the extinction advance angle is also derived accordingly, so as to avoid the negative effect of the asymmetric commutation voltage. Simulation experiments show that the new MQSS model proposed in this paper has higher simulation precision than the classical QSS model when asymmetric fault occurs in the AC system, by comparing both of them with the results of detailed electromagnetic transient (EMT) model of the DC transmission and its controlling system.
\end{abstract}

\section{Introduction}

By the end of 2013, China has built over 15 EHV and UHV DC transmission lines; both the total length and transmission capacity are the largest in the world [1]. These DC transmission systems are suitable to transmit large-scale renewable electric power generation [2-9] to the remote load center or link the energy storage [10] and electric vehicle-togrid [11] devices. Due to the intervention of large-scale DC transmission systems, especially the popular multiterminal VSC-based DC transmission [12-15], large amount of power electronic devices and other nonlinear elements have been introduced into the traditional AC power systems; the fast dynamic process of these components might increase the difficulty in performing the electromechanical transient simulation of hybrid AC/DC systems.

In order to improve the precision and speed of electromechanical transient simulation in hybrid AC/DC system, researchers have developed a variety of models for the DC system, including equivalent circuit model, dynamic phasor model, small signal linearized model, and classic quasi-steady state (QSS) model, for transient stability simulation.

The equivalent circuit DC system models mainly adopted the variable topology of converters, to establish the "centerprocess" method [16], Kron's method of tensor analysis by studying the state matrix of inverters [17], and the cut set matrix analysis method based on graph theoretical framework [18]. All the equivalent circuit based DC system models need a relatively large amount of computation for complicated operation states of thyristors. In order to save calculation time, other models such as the piecewise linearized model are used for DC system dynamic simulation [19]. However, these methods cannot be used in complex DC systems, such as bipolar or multiterminal DC systems, as it would be difficult to segment the complex converter topology.

Dynamic phasor method was firstly presented in [20]; its application for modeling of DC system, through Fourier series expansion, has been mainly used for harmonic analysis of hybrid AC/DC system [21-26]. 
Small signal linearized model was established by applying the actual sampling data to model the DC transmission system in the $d q$ reference frame $[19,27]$. It is able to take into account the nonlinear dynamic characteristics of the converters but only suitable for the small signal dynamic analysis, which cannot directly be transplanted to the electromechanical transient stability simulation problem.

Classical QSS model for the DC transmission and its controlling system has the advantage of fast computation; thus it has been widely used in hybrid AC/DC system simulation [28]. Although several techniques have been presented to improve the accuracy, such as adopting different step lengths during simulation [29], classical QSS model would still give wrong results under asymmetric faulted condition. To deal with the DC system modeling problem for transient stability simulation, a modified quasi-steady state (MQSS) model for DC system is established in this study.

The structure of the paper is as follows. The next section introduces the error causes for the classical QSS model. The modified QSS model is presented in Section 3; it uses the integral of the actual half-cycle voltage on the DC terminals and then calculates the average DC voltage, commutation angle, and the extinction advance angle accordingly. Effectiveness of the proposed model is tested on CIGRE HVDC benchmark system in Section 4, by comparing with the simulation results of EMT simulation software PSCAD/EMTDC. Finally, the conclusions are given in Section 5, which show that the proposed MQSS model has higher accuracy than classical QSS model, while increasing very limited computational complexity.

\section{Classic QSS Model of DC System}

The basic function of DC transmission system is to complete the AC to DC (rectifier) and DC to AC (inverter) conversion and transmission of electrical energy [30]. Taking the single bridge rectifier as an example, the connection topology of the converter system is shown in Figure 1. The single bridge converter has six bridge arms; each bridge arm is composed of one thyristor valve together with its triggering pulse control circuit. The most important advantage of DC system lies in its capability of controlling the converters' firing angles to adjust the operation mode of power systems very quickly.

2.1. Converter Bridge Model. This section describes the classical quasi-steady state model succinctly [31]. First of all, it is necessary to introduce the assumptions for classical QSS model as follows:

(1) The AC system is assumed to be three-phase symmetric sinusoidal system, with a frequency of $50 \mathrm{~Hz}$ $(60 \mathrm{~Hz}$ in other countries or regions), regardless of the harmonics and the influence of the neutral shift.

(2) The inductance value of the series smoothing reactor on DC side is large enough, and the performance of DC filters is ideal, so that the influence of the ripples can be neglected in the direct current.

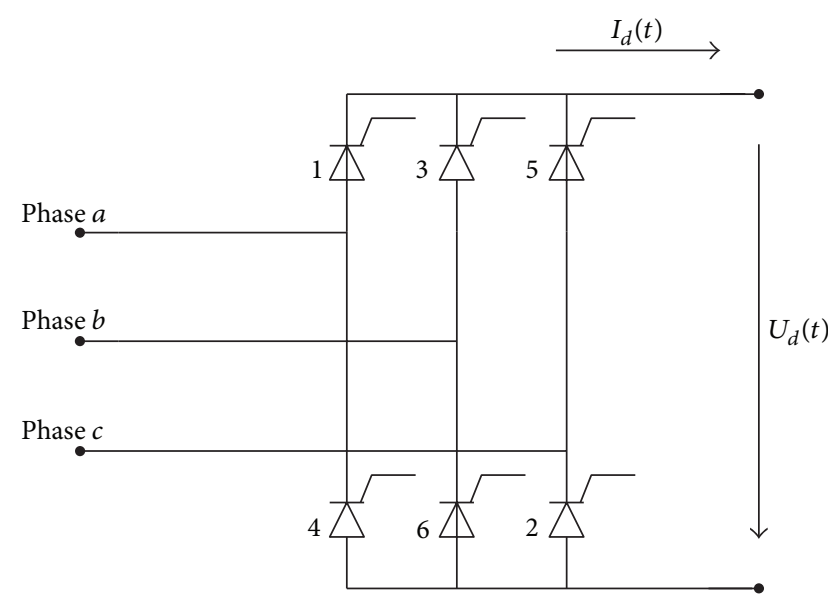

FIGURE 1: The principal wiring diagram of single bridge rectifier.

(3) The converter transformer is thought of as ideal, regardless of the saturation effect, excitation impedance and copper loss, and so forth.

(4) The characteristics of thyristor valves are ideal, namely, the voltage drop during conducting state and the leakage current during blocking state can be ignored, and the six valves are triggered to enter the conducting state in turn with an equal time interval of $1 / 6$ cycle.

Define the firing delay angle as $\alpha_{R}$ and commutation angle as $\mu_{R}$; then the DC voltage of both ends of the rectifier to the neutral point can be shown in Figure 2, where $e_{a}, e_{b}$, and $e_{c}$ denote the three-phase symmetric voltage on the AC side of rectifier and the symbol of $V_{i, j}$ means that valve $i$ commutates to valve $j$.

The instantaneous three-phase voltages can be expressed in (1), in which $U$ is the RMS value of phase voltage:

$$
\begin{aligned}
& e_{a}(\omega t)=\sqrt{2} U \sin (\omega t), \\
& e_{b}(\omega t)=\sqrt{2} U \sin \left(\omega t-\frac{2 \pi}{3}\right), \\
& e_{c}(\omega t)=\sqrt{2} U \sin \left(\omega t+\frac{2 \pi}{3}\right) .
\end{aligned}
$$

In classical QSS model, the average DC voltage can be directly calculated according to the symmetric three-phase commutation voltage waveform. Taking the commutation period of valve 3 to valve 4 (the shaded area in Figure 2), for example, the area of the shaded part can be seen as the average DC voltage of the rectifier bridge:

$$
U_{d R}=\int_{\alpha_{R}+5 \pi / 6}^{\alpha_{R}+7 \pi / 6}\left(e_{b}-e_{c}\right) d(\omega t)-\Delta V,
$$

where $\Delta V$ denotes the voltage drop caused by the commutation process, which can be generally expressed as the product of the equivalent commutation resistance $R_{R}$ and the DC current on rectifier side $I_{d R}$ (the subscript $R$ indicates 


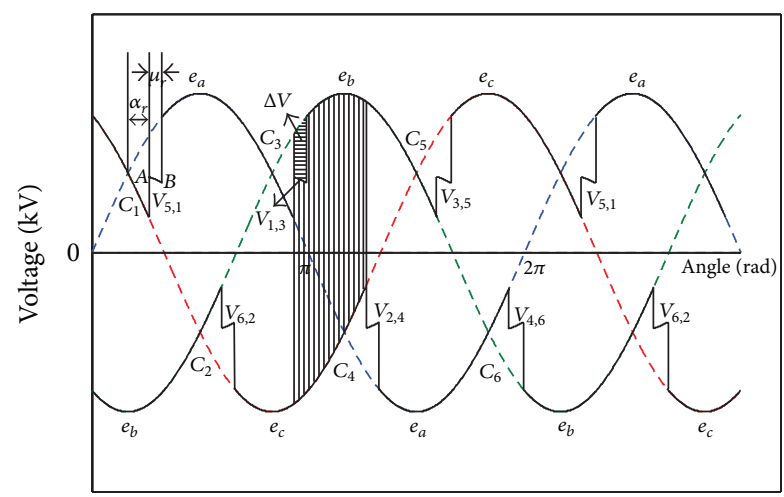

FIGURE 2: DC voltage on rectifier side under symmetric AC voltage waveform.

the variables are on the rectifier side; the subscript $I$ shows those belong to the inverter side).

Substitute (1) into (2):

$$
\begin{aligned}
U_{d R} & =\frac{3 \sqrt{2}}{\pi} E_{R} \cos \alpha_{R}-R_{R} I_{d R}, \\
R_{R} & =\frac{3 \omega L_{R}}{\pi},
\end{aligned}
$$

where $E_{R}$ is the RMS value of the commutation line voltage on rectifier side $/ \mathrm{kV}, I_{d R}$ is the DC current on rectifier side, $\alpha_{R}$ is the firing delay angle on rectifier side/rad, and $L_{R}$ is the equivalent commutation inductance of the rectifier side/ $\mathrm{H}$.

The commutation angle on rectifier side can be given as

$$
\mu_{R}=\cos ^{-1}\left(\cos \alpha_{R}-\frac{\sqrt{2} \omega L_{R}}{E_{R}}\right)-\alpha_{R}
$$

The RMS value of AC current on rectifier side will be

$$
I_{R}=\frac{\sqrt{6}}{\pi} I_{d R}
$$

The active power consumption by the converters and corresponding power factor on rectifier side can be written as

$$
\begin{aligned}
P_{R} & =P_{d R}=U_{d R} I_{d R}, \\
\cos \varphi_{R} & \approx \frac{1}{2}\left[\cos \alpha_{R}+\cos \left(\alpha_{R}+\mu_{R}\right)\right] .
\end{aligned}
$$

The formulas on inverter side of the DC transmission system are similar to the rectifier side in the classical QSS model; we only need to replace the variable of firing delay angle $\alpha_{R}$ with the extinction advance angle $\delta_{I}$.

2.2. DC Transmission Line Model. DC line model can be generally classified as lumped parameter circuit model, segmented $\pi$-type equivalent circuit model [32], Bergeron model based on distributed parameter [33], and frequencydependent circuit model [34], and so forth. The researchers can choose among these different DC line models, according

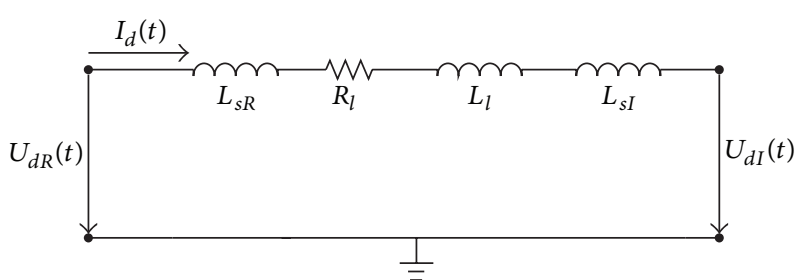

FIgURE 3: The equivalent circuit of the DC transmission lines.

to different accuracy requirements. In this study, it is mainly focused on the influence of asymmetric voltage on the firing angles of converters among different quasi-steady state models during electromechanical transient simulations; therefore, the DC transmission line model is selected as simple lumped parameter circuit model, such as the $R-L$ circuit shown in Figure 3.

According to Figure 3, it is easy to write the differential equation of DC transmission line during the electromechanical transient simulation as

$$
\left(L_{l}+L_{s R}+L_{s I}\right) \frac{d I_{d}(t)}{d t}+R_{l} I_{d}(t)=U_{d R}(t)-U_{d I}(t),
$$

where $R_{l}$ is the equivalent resistance of the DC transmission line $/ \Omega, L_{l}$ is the equivalent inductance of the DC transmission line/H, and $L_{s R}$ and $L_{s I}$ are the equivalent inductances of smoothing reactors on each side of the DC system $/ \mathrm{H}$.

2.3. DC Control System Model. The DC control system model is adopted as the CIGRE HVDC control system, the block diagram is shown in Figure 4, and it is easy to get the corresponding differential equation according to the transfer function of each block. The initial value of all state variables can be obtained from steady state power flow results. Combining the differential equations of both the control system and the DC transmission line equations, it is sufficient to solve the key parameters in DC systems, such as the firing delay angle $\alpha_{R}(t+\Delta t)$ and the extinction advance angle $\delta_{I}(t+$ $\Delta t)$, in which $\Delta t$ denotes the step length for time domain simulation.

2.4. Error Analysis of the Classical Quasi-Steady State Model. From the modeling of the three parts of DC systems from Sections 2.1 to 2.3, it can be seen that the classical QSS model only considers the situation when the commutation voltages are symmetric. In fact, during electromechanical transient simulation, the last three assumptions in Section 2.1 are easily satisfied, but the first assumption may not always be obeyed, because the AC bus voltage will no longer be symmetric during single phase or double phase short-circuit faults in the $\mathrm{AC}$ system. If we still use the symmetric waveform related formulas to fire the thyristors, it may bring serious deviation to the simulation results.

The asymmetric commutation voltage in the AC system may cause potential calculation errors for the classical QSS model in the following ways:

(1) If the average voltage of DC side is still computed according to formulas under symmetric voltage 


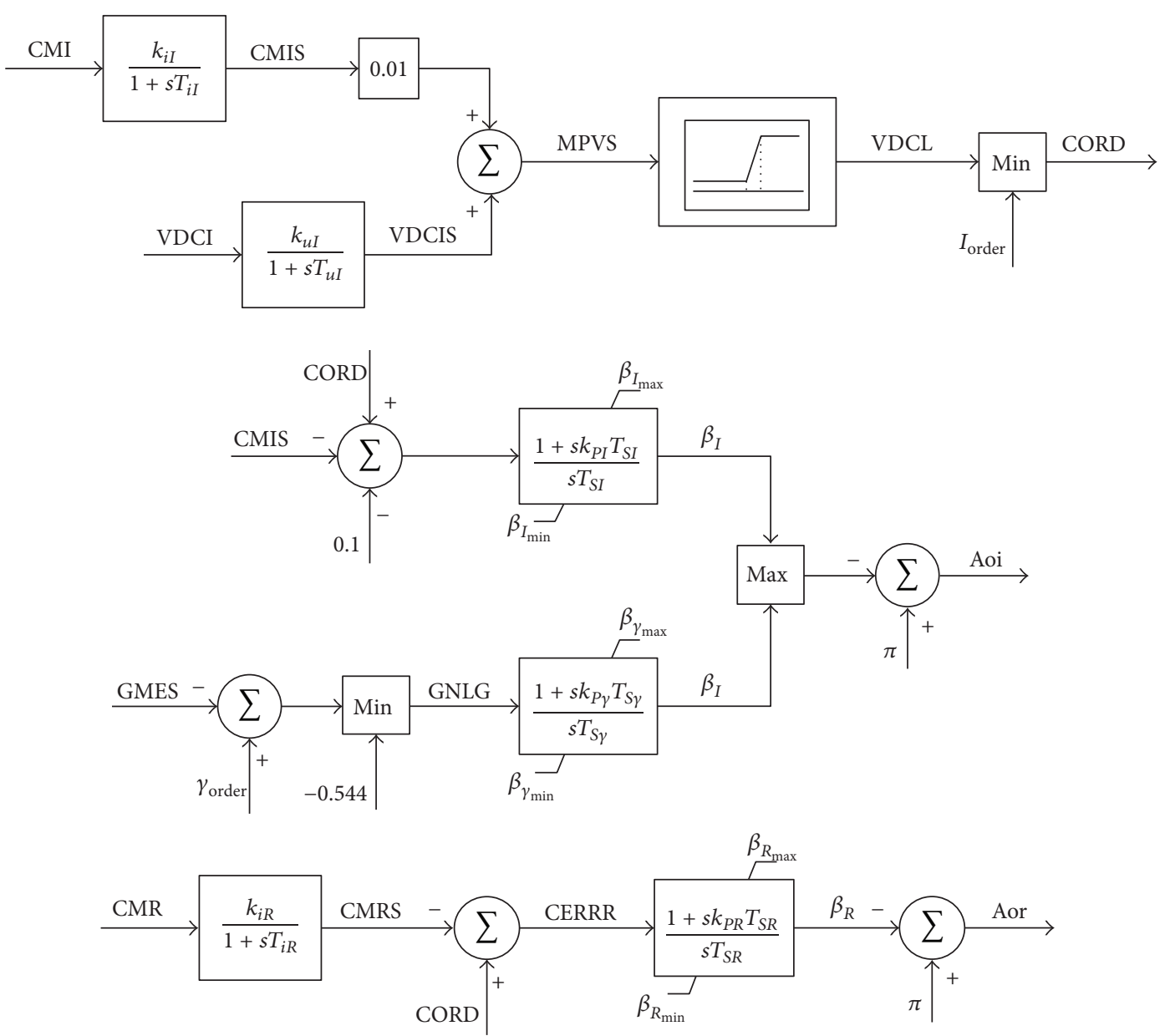

FIgURE 4: The control system of CIGRE HVDC test system.

assumption, all other parameters in the DC side will have inevitable deviation and thus affect the accuracy of calculation. Therefore, more precise formulas of the average DC voltage should be derived, according to the actual AC system operation status.

(2) If the rectifier firing delay angle and the inverter extinction advance angle are calculated using the symmetric waveform, the triggering pulse cannot consider the influence of the commutation voltage zero offset, which might lead to commutation failure or pole blocking for the DC system.

\section{The Modified QSS Model for the DC System}

It has been shown that the classical QSS model could not provide reliable simulation results under the condition of asymmetric commutation voltage. To address this main defect, a modified quasi-steady state model of DC system is proposed in this paper. It would be better to use the actual voltage waveform on the DC terminals to calculate the average DC voltage, so as to avoid the error caused by symmetric assumption. Before this, it should be better to find the exact commutation voltage zero point for the triggering pulse of each valve so as to compute the DC voltage and extinction advance angle. Taking the inverter side of six-pulse converter as an example, it is easy to illustrate the situation.

3.1. Exact Zero Point Prediction of Commutation Line Voltage. Since we only care about the fundamental components during electromechanical simulation, the influence of harmonics and interharmonics is not considered in this study. For a given operation status of three-phase voltage amplitude and phase angle, formulas can be derived for predicting the six zero points within one cycle; the detailed process is as follows.

Suppose the instantaneous three-phase asymmetric commutation voltages are expressed as

$$
\begin{aligned}
& e_{a}(\omega t)=\sqrt{2} U_{a} \sin \left(\omega t+\theta_{a}\right), \\
& e_{b}(\omega t)=\sqrt{2} U_{b} \sin \left(\omega t+\theta_{b}\right), \\
& e_{c}(\omega t)=\sqrt{2} U_{c} \sin \left(\omega t+\theta_{c}\right),
\end{aligned}
$$

using phase $a$ and phase $c$ to derive the prediction formula of the first line voltage zero point $C_{1}$. From (8), the line voltage $e_{a c}(\omega t)$ can be written as

$$
e_{a c}(\omega t)=\sqrt{2} U_{a} \sin \left(\omega t+\theta_{a}\right)-\sqrt{2} U_{c} \sin \left(\omega t+\theta_{c}\right) .
$$


At the line voltage zero point $C_{1}$, it should satisfy that $e_{a c}\left(C_{1}\right)=0$, according to (9):

$$
U_{a} \sin \left(C_{1}+\theta_{a}\right)=U_{c} \sin \left(C_{1}+\theta_{c}\right)
$$

Applying the trigonometric transformation, the zero point $C_{1}$ can be calculated by (12). Consider

$$
\begin{aligned}
& \sin \left(C_{1}\right)\left[U_{a} \cos \left(\theta_{a}\right)-U_{c} \cos \left(\theta_{c}\right)\right] \\
& =\cos \left(C_{1}\right)\left[U_{c} \sin \left(\theta_{c}\right)-U_{a} \sin \left(\theta_{a}\right)\right], \\
& C_{1}=\arctan \left(\frac{U_{c} \sin \theta_{c}-U_{a} \sin \theta_{a}}{U_{a} \cos \theta_{a}-U_{c} \cos \theta_{c}}\right) \pm \pi .
\end{aligned}
$$

The calculation formulas for the rest five line voltage zero points within one cycle can be acquired similarly, which are listed in

$$
\begin{aligned}
& C_{2}=\arctan \left(\frac{U_{b} \sin \theta_{b}-U_{c} \sin \theta_{c}}{U_{c} \cos \theta_{c}-U_{b} \cos \theta_{b}}\right) \pm \pi, \\
& C_{3}=\arctan \left(\frac{U_{a} \sin \theta_{a}-U_{b} \sin \theta_{b}}{U_{b} \cos \theta_{b}-U_{a} \cos \theta_{a}}\right) \pm \pi, \\
& C_{4}=C_{1}+\pi, \\
& C_{5}=C_{2}+\pi, \\
& C_{6}=C_{3}+\pi .
\end{aligned}
$$

3.2. Determination of Triggering Pulse. During the simulation process of asymmetric faults, due to the influence of the line voltage zero offset, phase locking device, and the DC control system, the actual firing delay angles for thyristor valves will not be equal to the initial angles given by the triggering pulse control system.

In order to address the influence of zero offset on the DC control system, the firing angle $\alpha$ by the control system is calculated by average value of the three adjacent zeros to determine a more accurate triggering pulse for each valve. This method is able to avoid the inaccurate triggering effect of classical QSS model during asymmetric faults. According to the exact commutation line voltage zero instants calculated by (12) and (13), triggering pulse of valve 3 can be acquired, and then the other five pulses can be obtained through the equidistant firing control as follows:

$$
\begin{aligned}
& p_{3}=\frac{\left(C_{1}+\alpha_{I}+2 \pi / 3+C_{2}+\alpha_{I}+\pi / 3+C_{3}+\alpha_{I}\right)}{3} \\
& p_{2}=p_{3}-\frac{\pi}{3} \\
& p_{1}=p_{2}-\frac{\pi}{3} \\
& p_{6}=p_{1}-\frac{\pi}{3} \\
& p_{5}=p_{6}-\frac{\pi}{3} \\
& p_{4}=p_{5}-\frac{\pi}{3}
\end{aligned}
$$

TABLE 1: The relationship between $p, q$, and $r$ and three-phase $a, b$, and $c$ for each triggering pulse.

\begin{tabular}{lllllll}
\hline Pulses & $p_{1}$ & $p_{2}$ & $p_{3}$ & $p_{4}$ & $p_{5}$ & $p_{6}$ \\
\hline$p$ & $a$ & $c$ & $b$ & $a$ & $c$ & $b$ \\
$q$ & $c$ & $b$ & $a$ & $c$ & $b$ & $a$ \\
$r$ & $b$ & $a$ & $c$ & $b$ & $a$ & $c$ \\
\hline
\end{tabular}

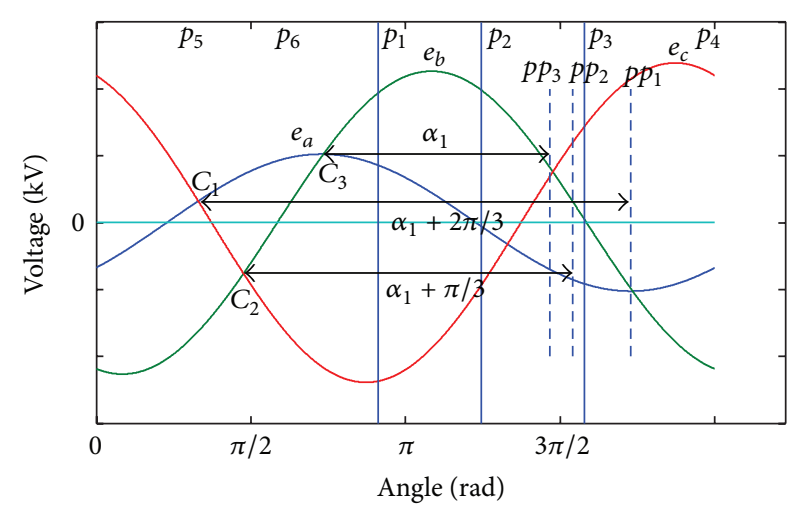

FIGURE 5: The determination of triggering pulse for the valves on the inverter side.

where $\alpha_{I}$ is the inherent firing delay angle on the inverter side that is initially set by the DC control system/rad.

Figure 5 shows the schematic diagram of triggering pulse of each valve on the inverter side, according to fault fundamental component of asymmetric commutation voltage by (8), where the parameters are chosen as $\alpha_{I}=2.3 \mathrm{rad}$, $U_{a}=51.249 \mathrm{kV}, U_{b}=113.268 \mathrm{kV}, U_{c}=119.422 \mathrm{kV}, \theta_{a}=$ $-0.7187 \mathrm{rad}, \theta_{b}=-1.8384 \mathrm{rad}$, and $\theta_{c}=-4.3136 \mathrm{rad}$.

It can be seen in Figure 5 that the three dashed lines of pulse $p p_{1}, p p_{2}$, and $p p_{3}$ are firing time instants for valve 3 by applying the three zeros $C_{1}, C_{2}$, and $C_{3}$, respectively. The solid line pulse $p_{3}$ is the average of these three firing angles, according to (14), and then the firing angles for other valves can be obtained by the equidistant firing control.

During the commutation process, three valves are participating; thus the valves can be divided into three classes. In this study, the valve that is entering into the commutation status is defined as $p$ phase, and exiting phase is defined as the $q$ phase, and the other half bridge that remains conducting is called the $r$ phase. For example, the relationship between $p$, $q$, and $r$ and original three-phase $a, b$, and $c$ for each triggering pulse plotted in Figure 5 can be shown in Table 1.

3.3. Average DC Voltage Calculation. Similar to the classical QSS model in Section 2.1, the average DC voltage under asymmetric faults can be calculated by the integral of actual commutation line voltage waveform on the DC terminals. Also, the voltage drop $\Delta V$ caused by DC current during the commutation process can still be replaced by the voltage drop on the equivalent commutation resistance.

According to Table 1, it is easy to obtain the entering, exiting, or conducting state of all the valves on the inverter side during the commutation period, and it is sufficient to 
calculate the exact DC voltage waveform and thus the DC voltage calculation formulas, which are shown in Table 2 . In Table $2, p_{i}$ denotes the firing angle for valve $i(i=1,2, \ldots, 6)$, and $\mu_{i}$ is the commutation angle of the valve $i$ after it has been triggered.

Using the last column of Table 2 to succinctly express the exact DC voltage waveform, then the average DC voltage can be calculated by the integral of six segments within one cycle:

$$
U_{d I}=-\frac{1}{2 \pi} \sum_{i=1}^{6}(-1)^{i-1}\left(\int_{p_{i}}^{p_{i+1}} e_{p r i}(\omega t) d \omega t-\Delta V\right),
$$

where $e_{\text {pri }}(\omega t)$ is the general DC voltage formula in the last column of Table 2 within each time period, $\Delta V$ is the voltage drop caused by commutation $/ \mathrm{kV}$, and $p_{i}$ is the electric angle of the triggering pulse for valve $i / \mathrm{rad}$.

Similar to classical QSS model, the voltage drop $\Delta V$ caused by commutation can also be calculated approximately through the voltage drop on equivalent resistance of the inverter side. Accordingly, the DC average voltage on the inverter side of the new MQSS model can be derived as follows:

$$
U_{d I}=-\frac{1}{2 \pi} \sum_{i=1}^{6}(-1)^{i-1}\left(\int_{p_{i}}^{p_{i+1}} e_{p r i}(\omega t) d \omega t\right)-R_{I} I_{d I} .
$$

Considering that the commutation voltage contains only the fundamental component, it is sufficient to integrate the average DC voltage by half-cycle voltage waveform. Taking the half-cycle voltage waveform from the triggering point of valve 6 to valve 3 of the inverter side as an example, the actual DC terminal voltage waveform on the inverter side can be shown as the shaded part in Figure 6, according to the expressions of DC voltages of Table 2 .

Then the average DC voltage from $p_{6}$ to $p_{3}$ can be calculated as

$$
\begin{aligned}
U_{d I} & =-\left\{\frac { 1 } { \pi } \left(\int_{p_{6}}^{p_{1}} e_{c b}(\omega t) d \omega t+\int_{p_{1}}^{p_{2}} e_{a b}(\omega t) d \omega t\right.\right. \\
& \left.\left.+\int_{p_{2}}^{p_{3}} e_{a c}(\omega t) d \omega t\right)-R_{I} I_{d I}\right\} .
\end{aligned}
$$

Solving the integral formula above, we can get the average DC voltage on the inverter side as shown in (18), under asymmetric commutation voltage:

$$
\begin{aligned}
U_{d I} & =-\left\{\frac { \sqrt { 2 } } { \pi } \left[U_{b}\left(\cos \left(p_{6}+\theta_{b}\right)-\cos \left(p_{5}+\theta_{b}\right)\right)\right.\right. \\
& -U_{c}\left(\cos \left(p_{6}+\theta_{c}\right)-\cos \left(p_{5}+\theta_{c}\right)\right) \\
& +U_{b}\left(\cos \left(p_{5}+\theta_{b}\right)-\cos \left(p_{4}+\theta_{b}\right)\right) \\
& -U_{a}\left(\cos \left(p_{5}+\theta_{a}\right)-\cos \left(p_{4}+\theta_{a}\right)\right) \\
& +U_{c}\left(\cos \left(p_{4}+\theta_{c}\right)-\cos \left(p_{3}+\theta_{c}\right)\right) \\
& \left.\left.-U_{a}\left(\cos \left(p_{5}+\theta_{a}\right)-\cos \left(p_{3}+\theta_{a}\right)\right)\right]-d_{I} I_{d I}\right\} .
\end{aligned}
$$

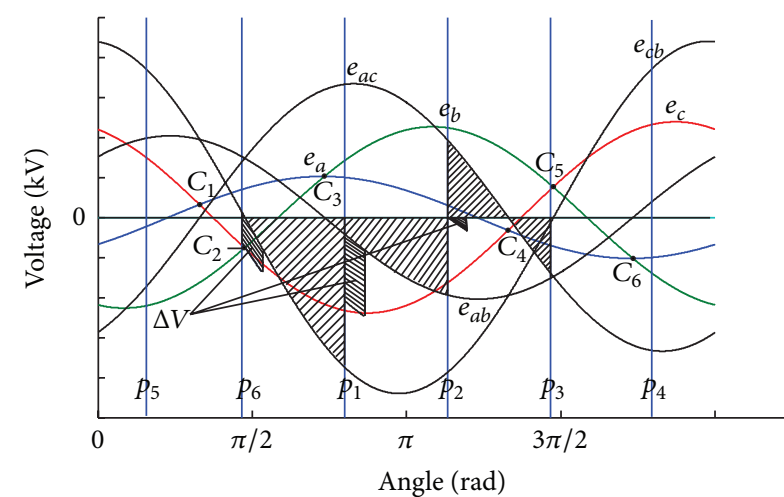

FIGURE 6: Actual DC voltage waveform analysis on the inverter side during asymmetric commutation line voltage.

The calculation process of average DC voltage on the rectifier side is similar. After obtaining the DC voltages on both sides of each DC system, other variables of DC system can then be calculated.

\section{Model Validation}

In order to test the validity of the proposed MQSS model, simulations are performed on CIGRE HVDC benchmark test system; according to the new model, the classical QSS model, and the power system EMT model from PSCAD software, results from PSCAD are chosen as the comparison reference, because it contains the full electromagnetic transient models that can take into consideration all dynamic performance of the DC system.

4.1. Three-Phase Symmetric Short-Circuit Fault on Inverter Side. At $1.0 \mathrm{~s}$, a three-phase symmetric short-circuit grounding fault occurs on the AC bus of the inverter side, the grounding resistance is set as $0 \Omega$, the fault lasts for $0.1 \mathrm{~s}$, and the total simulation time is $1.5 \mathrm{~s}$. With the same initial steady state and faulted operation conditions, the calculation results of the three models for the DC system, namely, the new modified quasi-steady state model (marked with "new"), the electromagnetic transient model (marked with "EMT"), and classical quasi-steady state model (marked with "QSS”), are shown in Figures 7-9. Among them, the ordinates are DC current of the inverter side, DC voltage on inverter side, and DC voltage on rectifier side in per unit system, and the abscissas are simulation time in seconds.

It can be seen from the curves of Figures 7-9 that the simulation results of both the new MQSS model (the solid blue curve) and the classical QSS model (the dotted green curve) match well with results of the EMT simulation (the dashed magenta curve), which demonstrates that both models are suitable to model three-phase faults since the commutation voltages are symmetric. The percent overshoots of the new MQSS model when the fault occurs and is being cleared are a bit more than the classical QSS model, because we use the measured half-cycle $\mathrm{AC}$ voltage waveform to calculate the $\mathrm{DC}$ variables, but the $\mathrm{AC}$ voltage waveform 


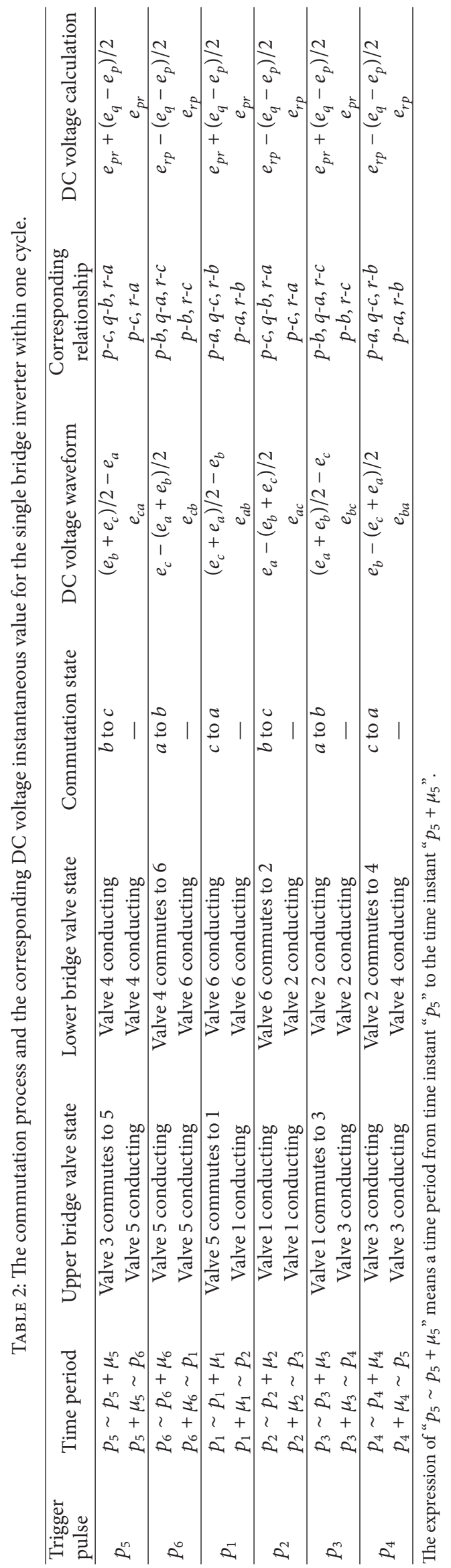


TABLE 3: Steady state DC values and errors during the $100 \mathrm{~ms}$ three-phase short-circuit fault for both QSS and MQSS models.

\begin{tabular}{cccccc}
\hline & EMT model $(\mathrm{pu})$ & QSS model $(\mathrm{pu})$ & Modified model $(\mathrm{pu})$ & $\begin{array}{c}\text { Absolute error } \\
\text { of QSS model }\end{array}$ & $\begin{array}{c}\text { Absolute error } \\
\text { of modified } \\
\text { model }\end{array}$ \\
\hline$I_{d}$ & 0.5500 & 0.5500 & 0.5500 & $\mathbf{0}$ & $\mathbf{0}$ \\
$U_{D I}$ & 0 & 0.0561 & 0.056 & $\mathbf{0 . 0 5 6 1}$ & $\mathbf{0 . 0 5 6 0}$ \\
$U_{D R}$ & 0.0050 & 0.0670 & 0.0670 & $\mathbf{0 . 0 1 7 0}$ & $\mathbf{0 . 0 1 7 0}$ \\
\hline
\end{tabular}

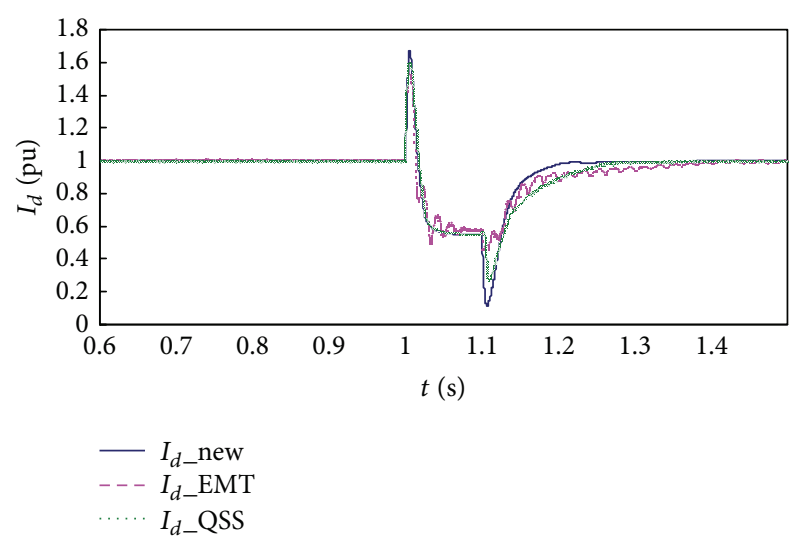

FIGURE 7: DC current transient waveform on the inverter side during three-phase grounding fault.

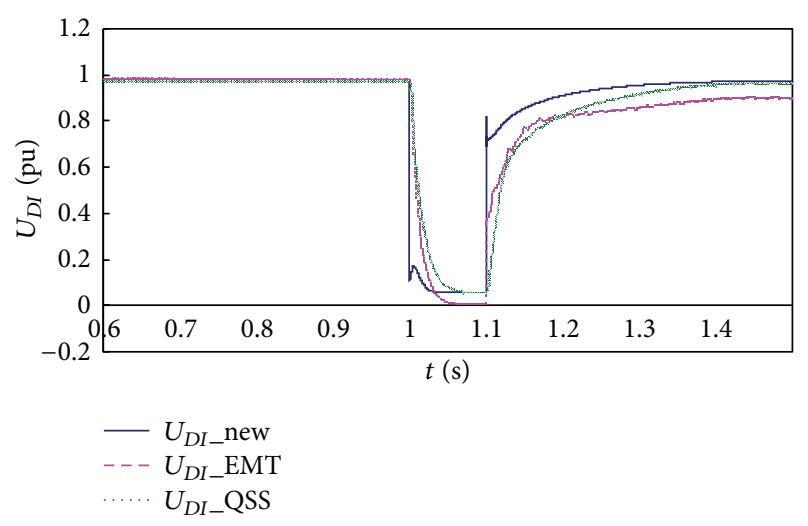

FIGURE 8: DC voltage transient waveform on the inverter side during three-phase grounding fault.

immediately after fault's occurrence and clearance is highly distorted. In electromechanical transient stability study, we care more about the AC fundamental components and the steady state DC values during and after faults; therefore, the comparison of steady state DC values during faults (from time $1.0 \mathrm{~s}$ to $1.1 \mathrm{~s}$ ) and corresponding errors of the two QSS models to EMT model are given in Table 3.

It can be seen from Table 3 that there is only slight differences between the calculation results of all three models; the steady state DC values during the $100 \mathrm{~ms}$ fault period for classical QSS model and new MQSS model are similar. This demonstrates that new MQSS model is reasonable and credible under symmetric commutation voltage condition,

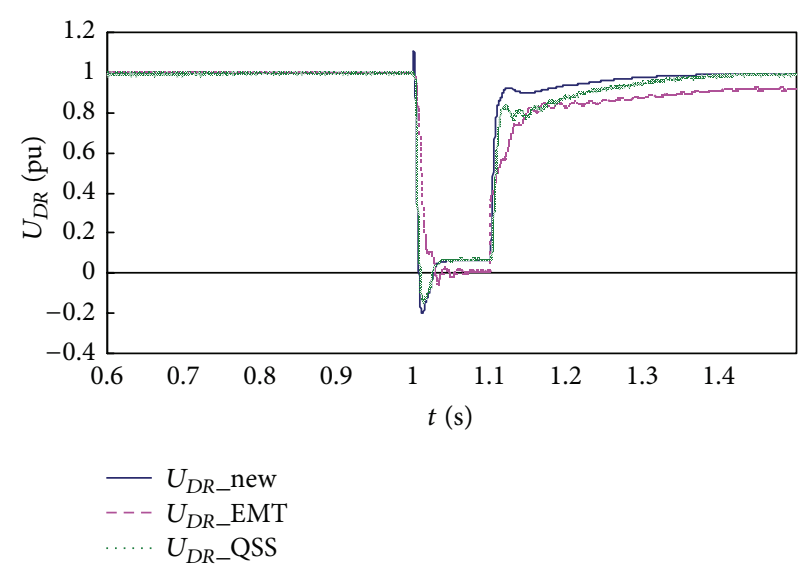

FIGURE 9: DC voltage transient waveform on the rectifier side during three-phase grounding fault.

although it utilizes the half-cycle measured faulted voltage waveform to predict the commutation voltage zero points and triggering pulse even during symmetric short-circuit faults.

4.2. Single Phase Grounding Fault on Inverter Side. Under asymmetric fault occurring on the AC bus of the inverter side, the voltage asymmetry is mainly decided by fault resistance. If the fault resistances are different, the asymmetric degree of the AC bus voltage will also be different. In this study, we use different values of the AC fault resistance under asymmetric faults to investigate the validity of the proposed MQSS model.

At $1.0 \mathrm{~s}$, a single phase grounding fault occurs on the AC bus of the inverter side, grounding resistance is $10 \Omega$, and the fault lasts for $0.1 \mathrm{~s}$, and the simulation results for the new model, EMT model, and classical QSS model are shown in Figure 10 to Figure 12.

It can be seen from Figures 10-12 that the calculation results of the new MQSS model are closer to EMT model than to the classical QSS model with same asymmetric fault conditions, although the percent overshoots of our MQSS model when the fault occurs and is being cleared are a bit more than the classical QSS model, which is caused by the fast controlling effect of the converters in the DC systems. The steady state values of the DC variables have higher accuracy using the new model, because it is able to address the zero point offsets and triggering pulse shifts during electromechanical transient stability simulation.

The comparison of steady state DC values during the single phase grounding fault (from time $1.0 \mathrm{~s}$ to $1.1 \mathrm{~s}$ ) and corresponding errors of the two QSS models to EMT model 
TABLE 4: Steady state DC values and errors during the $100 \mathrm{~ms}$ single phase grounding fault for QSS and MQSS models.

\begin{tabular}{|c|c|c|c|c|c|c|}
\hline $\begin{array}{l}\text { Single phase } \\
\text { grounding } \\
\text { resistance }(\Omega)\end{array}$ & Parameters & EMT model (pu) & QSS model (pu) & Modified model (pu) & $\begin{array}{c}\text { Relative error } \\
\text { of QSS model } \\
(\%)\end{array}$ & $\begin{array}{l}\text { Relative error of } \\
\text { modified model } \\
\text { (\%) }\end{array}$ \\
\hline \multirow{3}{*}{0} & $I_{d}$ & 0.5500 & 0.6000 & 0.5481 & 9.09 & 0.34 \\
\hline & $U_{D I}$ & 0 & 0.4267 & 0.1954 & - & - \\
\hline & $U_{D R}$ & 0.0507 & 0.4600 & 0.1843 & 807.30 & 263.51 \\
\hline \multirow{3}{*}{10} & $I_{d}$ & 0.5820 & 0.7338 & 0.5512 & 44.81 & 5.29 \\
\hline & $U_{D I}$ & 0.2929 & 0.5925 & 0.3331 & 102.29 & 13.72 \\
\hline & $U_{D R}$ & 0.3000 & 0.6139 & 0.3440 & 104.63 & 14.67 \\
\hline \multirow{3}{*}{20} & $I_{d}$ & 0.6823 & 0.8426 & 0.7164 & 23.49 & 5.00 \\
\hline & $U_{D I}$ & 0.5400 & 0.6929 & 0.5846 & 28.31 & 8.26 \\
\hline & $U_{D R}$ & 0.5600 & 0.7360 & 0.6001 & 34.73 & 7.16 \\
\hline
\end{tabular}

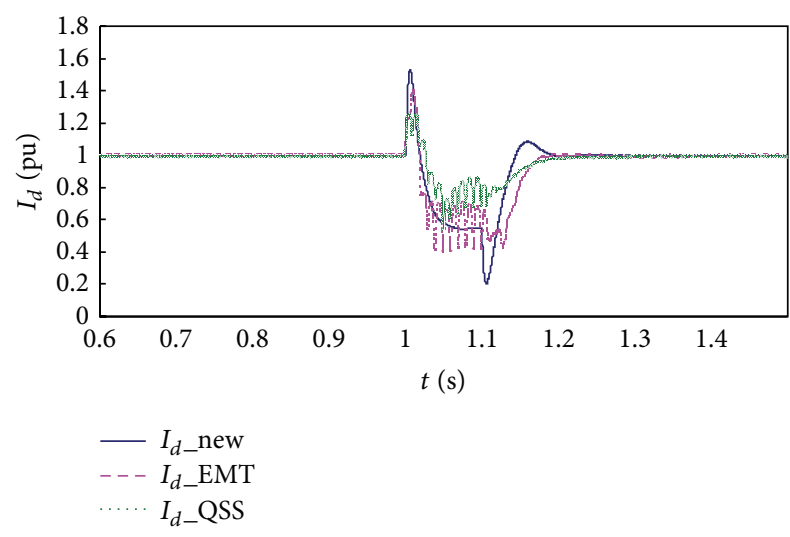

FIGURE 10: DC current transient waveform on the inverter side under single phase grounding fault.

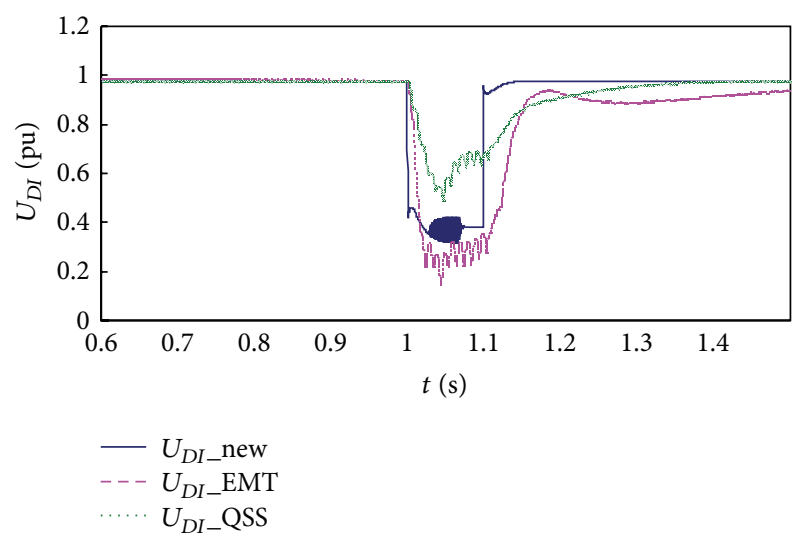

FIGURE 11: DC voltage transient waveform on the inverter side under single phase grounding fault.

are given in Table 4 . It can be seen from the data in Table 4 that the simulation errors of proposed new MQSS model are comparatively smaller than the classical QSS model, because the new model is able to consider the asymmetric commutation voltage under single phase grounding fault, and
TABLE 5: Simulation results for QSS and MQSS models under double phase-grounded faults with small grounding resistances.

\begin{tabular}{lccc}
\hline $\begin{array}{l}\text { Double } \\
\text { phase-grounded } \\
\text { resistance }(\Omega)\end{array}$ & EMT Model & $\begin{array}{c}\text { QSS model } \alpha_{I} \\
(\mathrm{rad})\end{array}$ & Modified model \\
\hline 0 & Blocking & Blocking & Blocking \\
20 & Blocking & 2.1817 & Blocking \\
40 & Blocking & 2.2689 & Blocking \\
\hline
\end{tabular}

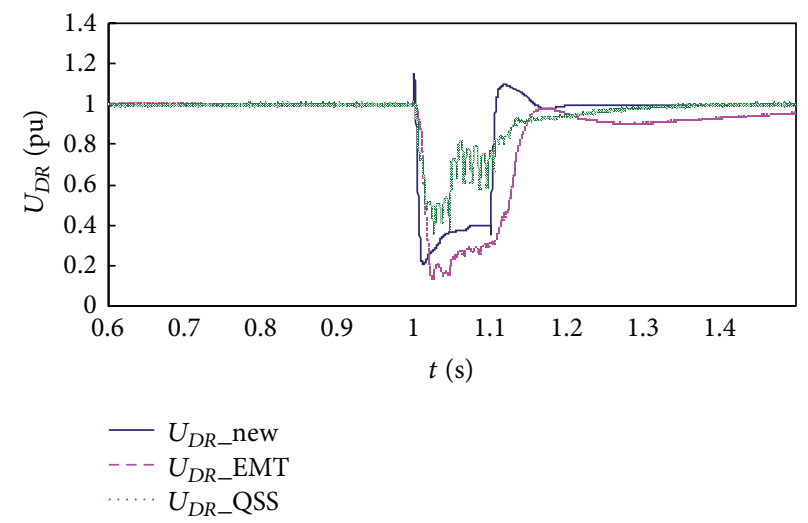

FIGURE 12: DC voltage transient waveform on the rectifier side under single phase grounding fault.

the deviations of classical QSS model to the EMT model are relatively larger. For example, when the grounding resistance is $10 \Omega$, the error rate of the calculated DC average voltage on the inverter side $U_{D I}$ during the $100 \mathrm{~ms}$ fault period by the new model has reduced by $88.57 \%$, comparing to the calculated value by the classical QSS model; the error rate of the DC average voltage on the rectifier side $U_{D R}$ is reduced by $89.96 \%$, and the error rate of the DC average current $I_{d}$ is reduced by $39.52 \%$. It demonstrates that the new modified quasi-steady state model has excellent ability to simulate the DC system under asymmetric faulted situation for electromechanical transient stability simulations. 
TABLE 6: Steady state DC values and errors during the $100 \mathrm{~ms}$ double phase-grounded fault for QSS and MQSS models.

\begin{tabular}{|c|c|c|c|c|c|c|}
\hline $\begin{array}{l}\text { Double } \\
\text { phase-grounded } \\
\text { resistance }(\Omega)\end{array}$ & Parameters & EMT model (pu) & QSS model (pu) & Modified model (pu) & $\begin{array}{l}\text { Relative error of } \\
\text { QSS model (\%) }\end{array}$ & $\begin{array}{c}\text { Relative error of } \\
\text { modified model } \\
(\%)\end{array}$ \\
\hline \multirow{3}{*}{60} & $I_{d}$ & 0.6500 & 0.8500 & 0.7470 & 30.77 & 14.92 \\
\hline & $U_{D I}$ & 0.4800 & 0.7400 & 0.6217 & 54.17 & 29.31 \\
\hline & $U_{D R}$ & 0.4750 & 0.7250 & 0.6380 & 52.63 & 34.32 \\
\hline \multirow{3}{*}{80} & $I_{d}$ & 0.7050 & 0.8840 & 0.7069 & 25.39 & 0.27 \\
\hline & $U_{D I}$ & 0.5700 & 0.7790 & 0.5733 & 36.67 & 5.79 \\
\hline & $U_{D R}$ & 0.5750 & 0.7800 & 0.6424 & 35.65 & 11.72 \\
\hline \multirow{3}{*}{100} & $I_{d}$ & 0.7900 & 0.9200 & 0.8104 & 16.46 & 2.58 \\
\hline & $U_{D I}$ & 0.6500 & 0.8000 & 0.6486 & 23.08 & 0.22 \\
\hline & $U_{D R}$ & 0.6520 & 0.8150 & 0.6623 & 25.00 & 1.58 \\
\hline
\end{tabular}

4.3. Double Phase-Grounded Fault on Inverter Side. Since double phase-grounded fault usually induces more severe asymmetry than phase-to-phase short-circuit, the phase-tophase short-circuit asymmetric fault type is not included in this study. At $1.0 \mathrm{~s}$, a double phase-grounded fault (assuming to be phase $a$ and phase $b$ ) occurs on the AC bus of the inverter side, and the fault lasts for $0.1 \mathrm{~s}$. Since the waveforms for the DC variables of the new model, EMT model, and classical QSS model are similar to the figures shown in Section 4.2, the figures are omitted, and the simulation results of the new MQSS model are closer to EMT model than to the classical QSS model under identical fault conditions.

Typically, the smaller the short-circuit grounding resistance, the lower the AC voltage; thus the commutation voltage asymmetry degree is larger. It is essential to simulate the more serious condition, such as commutation failure and pole blocking for the DC system.

In the accurate EMT simulation model, continuous commutation failure and HVDC pole blocking for the DC system can be encountered when the grounding resistance is from 0 to $40 \Omega$, with the initial firing delay angle of the inverter side $\alpha_{I}$ equal to $1.57 \mathrm{rad}$. However, no pole blocking phenomenon appears when the grounding resistance is 20 to $40 \Omega$ for the classical QSS model, and the pole blocking phenomenon can be reflected accurately by our new MQSS model, as shown in Table 5. It can be indicated from Table 5 that the modified QSS model is able to simulate the continuous commutation failure and pole blocking of the DC system during severely asymmetric AC faults; the simulation results are consistent with the accurate EMT model.

For larger grounding resistance from 60 to $100 \Omega$ under double phase-grounded fault, the simulation results and corresponding errors of the two QSS models to referenced EMT model are given in Table 6. It can be seen from the data in Table 6 that the simulation errors of new MQSS model proposed in this paper are comparatively smaller than the classical QSS model. During the $100 \mathrm{~ms}(1.0 \mathrm{~s}-1.1 \mathrm{~s})$ fault period, the relative errors of the average DC current and voltage variables by the new MQSS model are smaller than classical QSS model; the error can be reduced by $15 \%$ at least.
To conclude, the QSS-type models are developed for electromechanical transient simulation, which has much larger time steps and simulation duration than electromagnetic transient simulation. Therefore, the requirements are to reduce the amount of calculation time of electromagnetic computation, while maintaining high accuracy. It is well accepted that the detailed electromagnetic transient simulation programs, such as PSCAD/EMTDC, can provide the reference values; thus we compare both the proposed MQSS and the conventional QSS with the results of PSCAD. The accuracy of our MQSS model has shown to be substantially improved comparing with the conventional QSS model during asymmetric faults. And the computation time does not increase much, as indicated by the additional multiplications and additions in (12), (13), and (14) during the processes of zero point prediction and triggering pulse determination.

\section{Conclusion}

In power system electromechanical transient stability studies, the classical quasi-steady state (QSS) model is not able to accurately simulate the dynamic characteristics of DC transmission and its controlling system when asymmetric fault occurs in AC system; therefore, a new modified quasisteady state model (MQSS) is proposed in this paper. The new MQSS model utilizes the actual half-cycle voltage waveform on the DC terminals to predict the exact zero points of commutation voltages and then calculate the average DC voltages and the extinction advance angles, so as to avoid the negative effect of the asymmetric commutation voltage distortion. Simulation experiments show that the new MQSS model proposed in this paper can reduce the simulation error by $15 \%$ at least compared to the classical QSS model, under single phase grounding and double phase-grounded asymmetric faults in the AC system, by comparing both of the two models with the results of the detailed EMT model. Because the new MQSS model is capable of reflecting the dynamic characteristics of DC systems without considering the complicated electromagnetic transient processes in typical EMT models, it is very suitable for transient stability simulation in hybrid AC/DC power systems. 


\section{Conflict of Interests}

The authors declare that there is no conflict of interests regarding the publication of this paper.

\section{Acknowledgments}

This work was supported in part by China Postdoctoral Science Foundation under Grant 2013M542349, in part by the Fundamental Research Funds for the Central Universities of China under Grant xjj2013026, and in part by the State Key Laboratory of Electrical Insulation and Power Equipment under Grant EIPE14314.

\section{References}

[1] J. Cao and J. Cai, "HVDC in China," in Proceedings of the C-EPRI HVDC \& FACTS Conference, pp. 1-23, Beijing, China, August 2013, http://dsius.com/cet/HVDCinChina_EPRI2013_HVDC .pdf.

[2] D. Van Hertem and M. Ghandhari, "Multi-terminal VSC HVDC for the European supergrid: obstacles," Renewable and Sustainable Energy Reviews, vol. 14, no. 9, pp. 3156-3163, 2010.

[3] F. Chang, Z. Yang, Y. Wang, and S. Liu, "Fault characteristics and control strategies of multiterminal high voltage direct current transmission based on modular multilevel converter," Mathematical Problems in Engineering. In press.

[4] D. Niu, L. Ji, Q. Ma, and W. Li, "Knowledge mining based on environmental simulation applied to wind farm power forecasting," Mathematical Problems in Engineering, vol. 2013, Article ID 597562, 8 pages, 2013.

[5] A. Marucci, D. Monarca, M. Cecchini, A. Colantoni, A. Manzo, and A. Cappuccini, "The semitransparent photovoltaic films for Mediterranean greenhouse: a new sustainable technology," Mathematical Problems in Engineering, vol. 2012, Article ID 451934, 14 pages, 2012.

[6] J. Liu, W. Fang, X. Zhang, and C. Yang, "An improved photovoltaic power forecasting model with the assistance of aerosol index data," IEEE Transactions on Sustainable Energy, vol. 6, no. 2, pp. 434-442, 2015.

[7] G. Bergna, E. Berne, P. Egrot et al., "An energy-based controller for HVDC modular multilevel converter in decoupled double synchronous reference frame for voltage oscillation reduction," IEEE Transactions on Industrial Electronics, vol. 60, no. 6, pp. 2360-2371, 2013.

[8] D. Zhao, S. Meliopoulos, R. Fan, Z. Tan, and Y. Cho, "Reliability evaluation with cost analysis of alternate wind energy farms and interconnections," in Proceedings of the 44th IEEE North American Power Symposium (NAPS '12), pp. 1-6, IEEE, Urbana, Ill, USA, September 2012.

[9] J. Liu, G. M. Huang, Z. Ma, and Y. Geng, "A novel smart high-voltage circuit breaker for smart grid applications," IEEE Transactions on Smart Grid, vol. 2, no. 2, pp. 254-264, 2011.

[10] X. Hu, N. Murgovski, L. M. Johannesson, and B. Egardt, "Comparison of three electrochemical energy buffers applied to a hybrid bus powertrain with simultaneous optimal sizing and energy management," IEEE Transactions on Intelligent Transportation Systems, vol. 15, no. 3, pp. 1193-1205, 2014.

[11] X. Hu, N. Murgovski, L. Johannesson, and B. Egardt, "Energy efficiency analysis of a series plug-in hybrid electric bus with different energy management strategies and battery sizes," Applied Energy, vol. 111, pp. 1001-1009, 2013.

[12] S. Liu, Z. Xu, W. Hua, G. Tang, and Y. Xue, "Electromechanical transient modeling of modular multilevel converter based multi-terminal hvdc systems," IEEE Transactions on Power Systems, vol. 29, no. 1, pp. 72-83, 2014.

[13] G. O. Kalcon, G. P. Adam, O. Anaya-Lara, S. Lo, and K. Uhlen, "Small-signal stability analysis of multi-terminal VSC-based DC transmission systems," IEEE Transactions on Power Systems, vol. 27, no. 4, pp. 1818-1830, 2012.

[14] J.-H. Li, W.-L. Fang, Z.-C. Du, and D.-Z. Xia, "Calculation method of power flow in hybrid power system containing HVDC and FACTS," Power System Technology, vol. 29, no. 5, pp. 31-36, 2005.

[15] J. Beerten and R. Belmans, "Modeling and control of multiterminal VSC HVDC systems," Energy Procedia, vol. 24, pp. 123-130, 2012.

[16] N. G. Hingorani, J. L. Hay, and R. E. Crosbie, "Dynamic simulation of HVDC transmission systems on digital computers," Proceedings of the Institution of Electrical Engineers. IET Digital Library, vol. 113, no. 5, pp. 793-802, 1966.

[17] S. Williams and I. R. Smith, "Fast digital computation of 3phase thyristor bridge circuits," Proceedings of the Institution of Electrical Engineers, vol. 120, no. 7, pp. 791-795, 1973.

[18] K. R. Padiyar, "Digital simulation of multiterminal HVDC systems using a novel converter model," IEEE Transactions on Power Apparatus and Systems, vol. 102, no. 6, pp. 1624-1632, 1983.

[19] C. M. Osauskas, D. J. Hume, and A. R. Wood, "Small signal frequency domain model of an HVDC converter," IEE Proceedings-Generation, Transmission and Distribution, vol. 148, no. 6, pp. 573-578, 2001.

[20] C. Demarco L and C. Verghese G, "Bringing phasor dynamics into the power system load flow," in Proceedings of the 25th North American Power Symposium, pp. 31-38, Washington, DC, USA, October 1993.

[21] S. Cole, J. Beerten, and R. Belmans, "Generalized dynamic VSC MTDC model for power system stability studies," IEEE Transactions on Power Systems, vol. 25, no. 3, pp. 1655-1662, 2010.

[22] S. Cole and R. Belmans, "A proposal for standard VSC HVDC dynamic models in power system stability studies," Electric Power Systems Research, vol. 81, no. 4, pp. 967-973, 2011.

[23] C. Hahn, A. Semerow, M. Luther, and O. Ruhle, "Generic modeling of a line commutated HVDC system for power system stability studies," in Proceedings of the IEEE PES on T\&D Conference and Exposition, pp. 1-6, IEEE, Chicago, Ill, USA, 2014.

[24] H. Zhu, Z. Cai, H. Liu, Q. Qi, and Y. Ni, "Hybrid-model transient stability simulation using dynamic phasors based HVDC system model," Electric Power Systems Research, vol. 76, no. 6-7, pp. 582-591, 2006.

[25] D. A. Woodford, A. M. Gole, and R. W. Menzies, "Digital simulation of dc links and ac machines," IEEE Transactions on Power Apparatus and Systems, vol. 102, no. 6, pp. 1616-1623, 1983.

[26] J. Reeve and S. P. Chen, "Digital simulation of a multiterminal HVDC transmission system," IEEE Transactions on Power Apparatus and Systems, vol. 103, no. 12, pp. 3634-3642, 1984.

[27] C. Osauskas and A. Wood, "Small-signal dynamic modeling of HVDC systems," IEEE Transactions on Power Delivery, vol. 18, no. 1, pp. 220-225, 2003. 
[28] Y.-X. Ni, V. Vittal, W. Kliemann, and A. A. Found, "Nonlinear modal interaction in hvdc/ac power systems with dc power modulation," IEEE Transactions on Power Systems, vol. 11, no. 4, pp. 2011-2017, 1996.

[29] R. M. Brandt, U. D. Annakkage, D. P. Brandt, and N. Kshatriya, "Validation of a two-time step HVDC transient stability simulation model including detailed HVDC controls and DC line L/R dynamics," in Proceedings of the IEEE Power Engineering Society General Meeting, pp. 1-6, IEEE, Montreal, Canada, July 2006.

[30] V. K. Sood, HVDC and FACTS Controllers: Applications of Static Converters in Power Systems, Springer Science \& Business Media, New York, NY, USA, 2004.

[31] N. Rostamkolai, A. G. Phadke, W. F. Long, and J. S. Thorp, "An adaptive optimal control strategy for dynamic stability enhancement of AC/DC power systems," IEEE Transactions on Power Systems, vol. 3, no. 3, pp. 1139-1145, 1988.

[32] M. Khatir, S. A. Zidi, M. K. Fellah, S. Hadjeri, and O. Dahou, "HVDC transmission line models for steady-state and transients analysis in SIMULINK environment," in Proceedings of the 32nd Annual Conference on IEEE Industrial Electronics (IECON '06), pp. 436-441, IEEE, Paris, France, November 2006.

[33] F. E. Menter and L. Grcev, "EMTP-based model for grounding system analysis," IEEE Transactions on Power Delivery, vol. 9, no. 4, pp. 1838-1847, 1994.

[34] J. R. Marti, "Accurate modelling of frequency-dependent transmission lines in electromagnetic transient simulations," IEEE Transactions on Power Apparatus and Systems, vol. PAS-101, no. 1, pp. 147-157, 1982. 


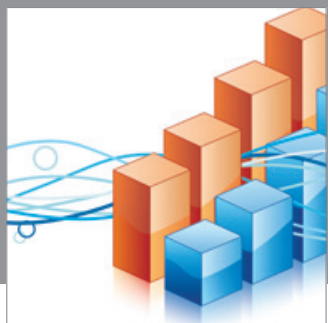

Advances in

Operations Research

mansans

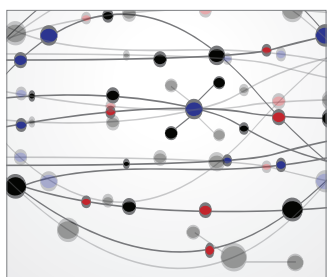

The Scientific World Journal
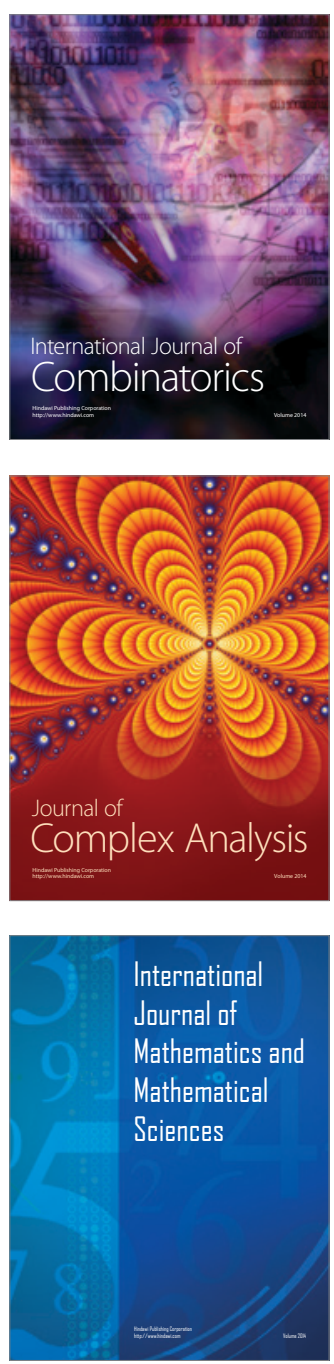
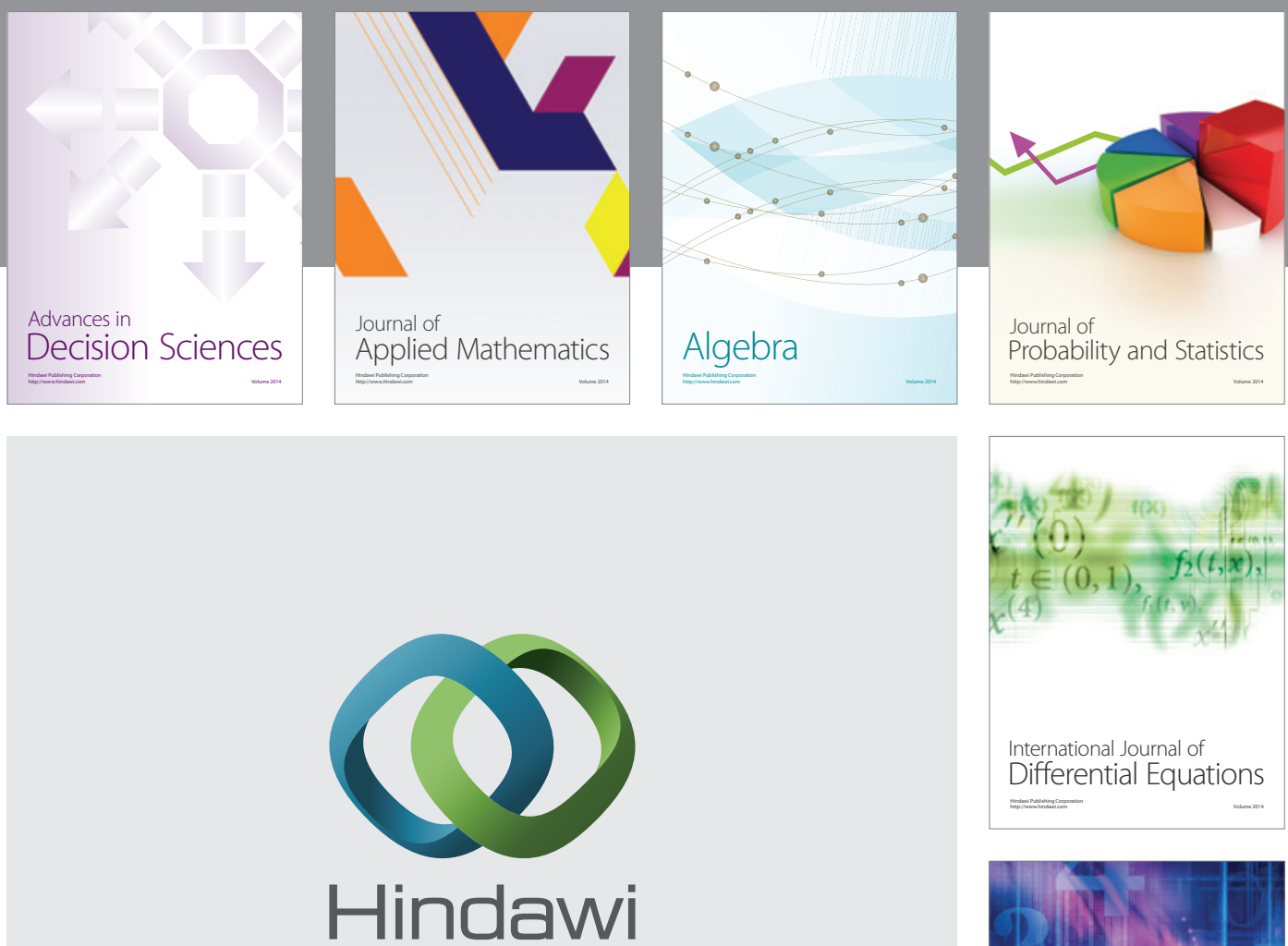

Submit your manuscripts at http://www.hindawi.com
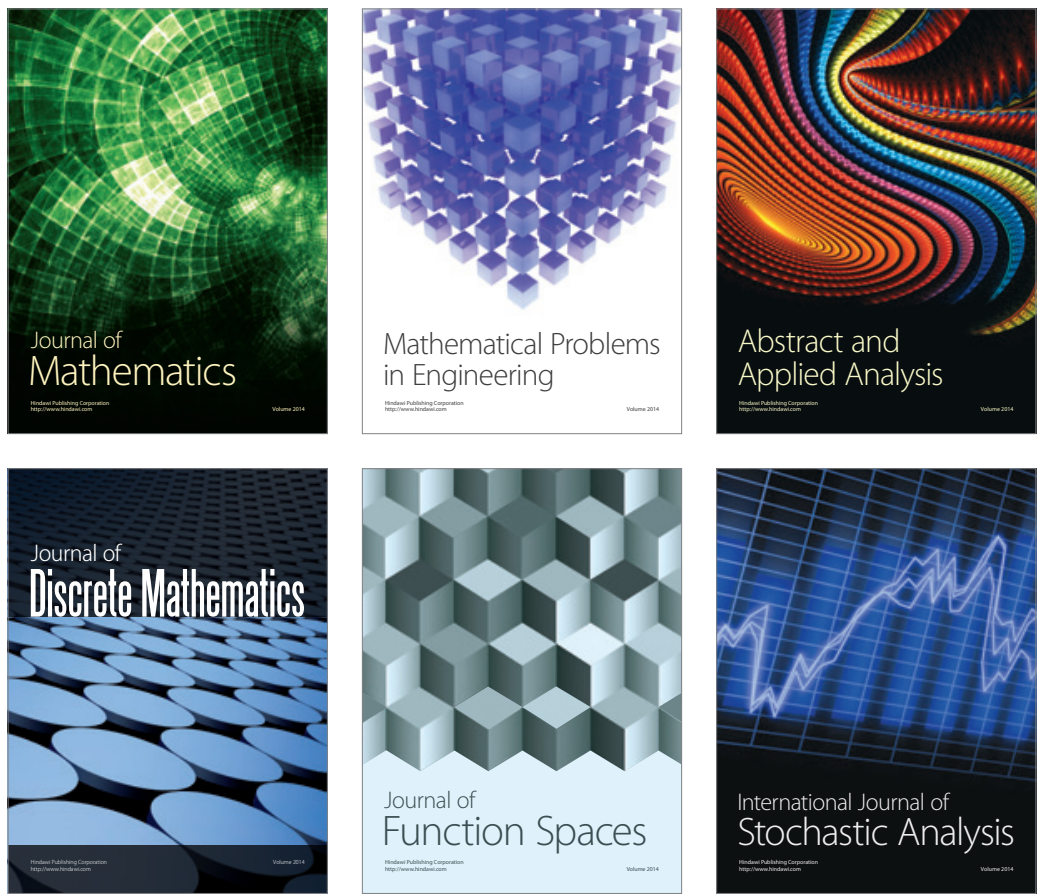

Journal of

Function Spaces

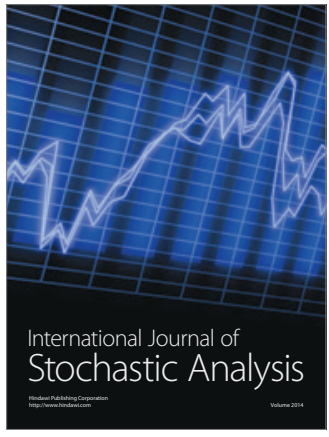

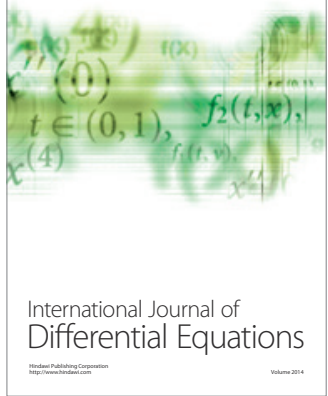
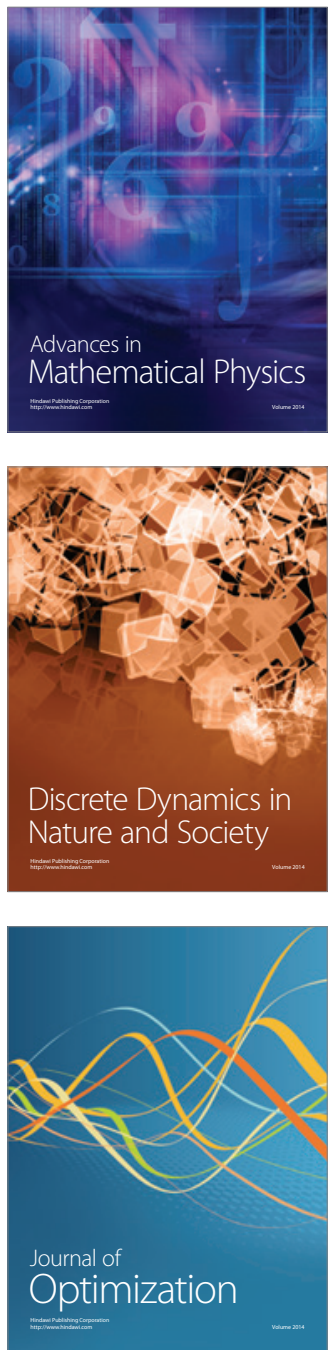